\title{
Gender Differences in Adolescent Neuroticism
}

\author{
Brinda B. Sharma \\ Lecturer( Applied Psychology) Dibrugarh University, Assam
}

\begin{abstract}
Societal sphere of adolescence-family milieu and peer context are important in terms of their normative and positive advancement. Appearance anxiety affects the attitudes and behaviours of both males and females but gender often temperate the strength of these relationships. The aim of this research is to examine the influence of perception of individual attractiveness and interpersonal relationship (with parents) on neuroticism in adolescent boys and girls. A sample of 100 adolescents selected randomly (Age-16-18 years) from different schools in Dibrugarh were considered for the study. Findings of the study revealed significant correlation of both the variables on neuroticism in adolescents. The correlation was negative for a cordial level of parent child relationship and positive for the remaining variable. Gender differences were noted in the degree of neuroticism as well as in appraisal of self and stress tolerance in interpersonal relations. In the context of the present changing social dynamics, the results of the present study have implications for both individual counseling as well as for the inherent discrimination in the process of socialization. In the former context it implies the need to sensitize girls and boys to the importance of self acceptance and positivity in attitude and appraisal of self and others to develop into well adjusted individuals. In the latter context it implies the significance of a non-discriminating socialization process for both boys and girls where parents encourage their daughters to develop a positive self-image and a hardy personality.

Keywords: Adolescence, Gender, Neuroticism, Socialization, Symbolic Interactionism.
\end{abstract}

\section{Introduction}

Change characterizes human condition and at no phase in human development, apart from the first two years of life, is change more pervasive and rapid than in adolescence. Everything changes and along with the emotional, physical and cognitive changes in the self noted by the adolescent, the world around him/her also changes in many respects. This is especially true regarding their perceptions of parental control and family dynamics as well as in their equations with their peers. Sociocultural norms pertaining to appearance encourage women to engage in potentially harmful behaviours such as eating disorders, cosmetic surgery and intentional sunbathing to a larger degree than men (Scot Burton, Richard Netemeyer, Lichtenstein, 1995). Thus the rationale of the present study is to understand the differentialperceptions and expectations associated with boys and girls in the context of society and family. It is postulated that the gender based differences in perception and attitude towards one's own physical attractiveness along with the level of cordiality in parent-child and peer relationships will have an effect on the individual's neurotic tendencies. The researcher addresses the following research questions-

(1) Is there a relationship between the individual's physical attractiveness and his/her level of neuroticism?

(2) Does parent-child relationship pattern differ among adolescent boys and girls?

(3) Is there a relationship between the individual's degree of interpersonal stress and his/her degree of neuroticism?

(4) Does neuroticism differ significantly among adolescent girls and boys?

The Researcher then addresses the implications of the present study on psychological constructs like depression, anxiety, adjustment, self-concept, achievement motivation and overall psychological well-being in females and on the significance of sensitization of both girls and boys on issues of gender discrimination and stereotypes. Women have higher rates of negative affect and depression and poorer subjective health than men (Nydegger, 2004; Russo \& Green, 1993). The researcher further focuses on the importance of a cordial parent-child relationship to encourage assertiveness, coping skills and self-acceptance especially among girls.

It is accepted that gender crosses all bounds of "Race, Class and Place" (Brown, 1990) and is interwoven in our identity as individuals (Goldner, 1985). The lives of women have progressed in many objective measures such as educational accomplishment, reduction of gender pay gap etc. However empirical research shows that the measures of subjective well-being among women have declined both absolutely and relative to men (Stevenson, Wolfers, 2009). There is the commonly held view that families consider it less important to educate a girl than a boy as boys are seen as breadwinners of the family. Girls are expected to get married, play the roles of a house-wife and mother and maybe take up a job that is complementary to these roles. Powerful conventions within the family and the broader society have tended to govern their aspirations 
and the choices that will realize them. Bounded by such norms of socialization, neuroticism or anxiety is seen more among females than males particularly to match the expectations related to their marriage and in their interpersonal relationships. It is assumed that neuroticism is a continuum with extremely adjusted or so-called normal behaviour at one end and extremely maladjusted behaviour to the extent of pathogenic problems at the other end (Kundu, 1996). The ideological conventions of gender and sexuality are studied early in the socialization progression, ensconced firmly during adolescence and conveyed in large part by the popular culture.

Man is essentially a symbol using creature and survives in a world created by the use of these symbols. The result of this state is that man's existence is mediated as well as created by symbols. This is the core concept of the theory of Symbolic Interactionism which is referred in the present study in the context of assigning the construct of beauty as a learnt symbol of feminity. As these symbols are learnt they are prone to be unlearnt as well. According to Baldwin (1897), Cooley (1902) and Mead (1925, 1934), the self is socially constructed through the linguistic exchanges or symbolic interactions with significant others. Thus they stressed that the personal self is formed by the symbolic interactions with significant others like family and peers. In the present paper an attempt is made to understand the parent child relationship level as well as interpersonal stress owing to this relationship among the sample adolescents considered for the study and whether the interactions among the sample adolescents considered for the study and whether the interactions among these significant others have an association with the personality construct of neuroticism.

Socialization is the route by which individuals acquire the familiarity, speech, social skills and values to conform to the norms and roles required for integration to a group or community. The way people are handled as children have a great effect on certain aspects of their later personality. Parents or primary care givers and peers are the most important socializing agents for an adolescent. Warmth and acceptance on the part of parents lead to a number of desirable personality traits, including conscience, sympathy and affiliative motivation (Michael Argyle, 1964). Peer culture is an important socialization variable which refers to a constant set of activities, values, objects and concerns that children create and allocate in interaction with peers. Most research on peer culture has focused on adolescent peer values identities and interests (Coleman,1961; Cusick, 1972; Blyth \& Simmons,1987). The symbolic interaction with peers is correlated with the formation of self-concept and identity in adolescents. Empirical studies show gender differences in adolescents' reactivity to daily interpersonal events as well in the strength of daily mood as a predictor of interpersonal events. Self-acceptance and hardiness in adolescents has implications on his/her everyday functioning and long-term adjustment.

On the basis of the above discussion three null hypotheses were set:

H1: Level of Neurosis would not vary with gender.

$\mathrm{H} 2$ : There would be no significant correlations among ratings of individual attractiveness and Neuroticism in adolescent girls.

H3: There would be no significant correlations among pattern of parent-child relationship and Neuroticism in adolescent girls and boys.

\section{Sample}

\section{Methodology}

The participants were 100 Higher Secondary students (Girls=50, Boys=50) from 2 Higher Secondary school classes from Dibrugarh, Assam. The mean age of the total sample was 16.8 years. Incidental random sampling technique was employed and only those adolescents who were living with both their parents and studying in regular schools were selected.

\section{Tools}

The following tools were employed to study the variables of the study:

Kundu Neurotic Personality Inventory (KNPI, 1987) developed by Dr. Ramanath Kundu was used to assess the potential neurotic tendency in adolescent boys and girls. The KNPI is a 66 item five-point rating scale that yields a total score and classifies the sample into four groups viz. Normal, Slightly Neurotic, Moderately Neurotic and Highly Neurotic. Reliability of the scale was determined by the split-half method. The odd-even reliability coefficient was computed as 0.82 whereas the 1 st half- 2 nd half coefficient was 0.88 . The validity coefficient was computed as 0.86 .

Parent-Child Relationship Questionnaire (PCRS-RN) developed by Late Nalini Rao was used to study parent- child relationship dimensions. The tool contains 100 items categorized into ten dimensions namely, protecting, symbolic punishment, rejecting, object punishment, demanding, indifferent, symbolic reward, loving, object reward and neglecting. The operational definitions of the dimensions are as follows: Demanding is an expression of authority and claim over the child, Indifferent is the expression of unconcerned, passive behaviour indicating disinterest in the child, loving is the expression of devoted attachment shown to the child, Neglecting is a careless slighting treatment coupled with deliberate disregard towards the child, Protecting is a 
defending attitude, sheltering the child from potential threat, Rejecting is renouncing the child in aversion, Symbolic/Object Reward indicate parents' acceptance of the child while Symbolic/Object Punishment is a means to show parents' annoyance with the child. Each respondent score the tool for both father and mother separately. The PCRS-RN is a five point rating scale ranging from 'Always' to 'Very Rarely'. The test-retestreliability coefficient ranged from .770 to .871 over the ten sub-scales. Face validity of the scale was already established at the development stage and the construct validity of the scales ranged from .289 to .578 was found to be significant at .05 levels or above.

A self-rating of the adolescents were obtained for physical attractiveness where the subjects rated their physical attractiveness on a five-point scale ranging from "very good looking" to "very plain looking". The distribution of the ratings of adolescent males and females are given in Table1.

\section{Procedure}

Data was collected on the basis of a pre-arranged programme scheduled with the respective schools. A rapport was established with the subjects and after obtaining their consent the subjects were instructed to be honest in their responses to the questions. The purpose of the study was partially explained though the term 'neurotic' was deliberately avoided to minimize test anxiety. The data was collected by administering the KNPI on small groups of 15-20 high school students and by administering the PCRS-RN on the same after a break of one hour duration. The data were scored with the help of standard scoring keys and scores were tabulated and treated statistically by using descriptive statistical methods.

\section{Results And Discussion}

Table I

Self rating of physical attractiveness of adolescents (males and females)

\begin{tabular}{|c|c|c|c|c|}
\hline \multirow{2}{*}{$\begin{array}{c}\text { Ratings of Physical } \\
\text { Appearance }\end{array}$} & \multicolumn{3}{|c|}{ Males } & \multicolumn{2}{c|}{ Females } \\
\cline { 2 - 5 } & No. & Percentage & No. & Percentage \\
\hline Very Good Looking & 12 & 24 & 11 & 22 \\
\hline Good Looking & 22 & 44 & 25 & 50 \\
\hline Fairly Good Looking & 10 & 20 & 4 & 8 \\
\hline Plain Looking & 6 & 12 & 6 & 12 \\
\hline Very Plain Looking & 0 & 0 & 4 & 8 \\
\hline Total & 50 & 100 & 50 & 100 \\
\hline
\end{tabular}

The reliability of the self ratings as judged by their correlations with repeat ratings made after 4 months of the original ones was .54 and .66 for adolescent males and females respectively. The above table1 shows that female adolescents are more prone to negative self-evaluations as indicated by the percentage of females who rate themselves as very plain in their physical attractiveness. This is corroborated by the findings of Ernest W. Burgess and Paul Wallin (1944). In the present study the self-ratings are not compared with the ratings of peers and significant others which can be explored in a further study.

Table II

Mean and Standard Deviation of Neuroticism in Adolescent Males and Females

\begin{tabular}{|c|c|c|}
\hline Gender & Mean & SD \\
\hline Males & 2.23 & 0.54 \\
\hline Females & 5.35 & 0.79 \\
\hline
\end{tabular}

As seen in Table II, mean neuroticism in adolescent females is slightly higher than the mean neuroticism in adolescent males. Persons scoring high in neuroticism may be depicted succinctly as having ups and downs in disposition, easily upset, subject to melancholy, awkward in public and withdrawn in intimate relationships, lacking in self-confidence and having a tendency to day dream. Neuroticism is linked with happiness in the study conducted by Vitters and Nilsen (2002) and the absence of the above characteristics may be an indicator of subjective well-being. The findings specified in Table II have significant implications for the adjustment in adolescent girls. Thus the first hypothesis (H1) that level of neuroticism would not vary with gender is rejected. The extent of this variation can be undertaken in a separate study. 
Table III

Correlation of ratings of physical appearance and level of neuroticism in adolescent females

\begin{tabular}{|c|c|c|c|c|}
\hline \multirow{2}{*}{$\begin{array}{c}\text { Rating of } \\
\text { Appearance }\end{array}$} & Normal & Slightly Neurotic & $\begin{array}{c}\text { Moderately } \\
\text { Neurotic }\end{array}$ & Highly Neurotic \\
\cline { 2 - 5 } & 0.12 & 1.04 & 0.87 & 0.23 \\
\hline $\begin{array}{c}\text { Very Good } \\
\text { Looking }\end{array}$ & $3.24^{* *}$ & 0.98 & 1.01 & 0.18 \\
\hline $\begin{array}{c}\text { Good Looking } \\
\text { Fairly Good } \\
\text { Looking }\end{array}$ & $4.63^{*}$ & 2.16 & $3.03^{* *}$ & 2.27 \\
\hline Plain Looking & 1.69 & 2.02 & $3.43^{* *}$ & 2.94 \\
\hline $\begin{array}{c}\text { Very Plain } \\
\text { Looking }\end{array}$ & 1.23 & 1.54 & $4.01^{* *}$ & 1.98 \\
\hline
\end{tabular}

**Significant at .05 level of significance *Significant at .01 level of significance

The above Table III indicates the correlation between ratings of physical appearance and neuroticism in adolescent females. Adolescent males and females vary in neurotic tendencies which indicate a difference in their susceptibility to negative emotions and general distress. The hypothesis that physical attractiveness would correlate positively with happiness and self-esteem and negatively with neuroticism was supported for women but not for men (Eugene W. Mathes \& Arnold Kahn (1975): Physical Attractiveness, Happiness, Neuroticism, and Self-Esteem, The Journal of Psychology: Interdisciplinary and Applied, 90:1, 27-30 ).Significant positive correlations $(\mathrm{p}<0.05)$ between moderate neuroticism and negative self rating of physical appearance among adolescent females were noted. Physical appearance or attractiveness as an input in heterosexual exchange is more valuable for women than for men. Thus it follows that the correlation of physical appearance with psychological health (neuroticism) and self acceptance is more significant for women than for men. The above discussion thus enables the researcher to reject the second hypothesis $(\mathrm{H} 2)$ that there would be no significant correlations among ratings of individual attractiveness and neuroticism in adolescent girls.

Table IV

Correlation between the ten dimensions of parent-child relationship and neuroticism in adolescent males and females

\begin{tabular}{|c|c|c|}
\hline Dimensions & Neuroticism & Neuroticism \\
\hline Protecting & -0.22 & $-0.35^{* *}$ \\
\hline Symbolic Punishment & 0.18 & $0.27^{* *}$ \\
\hline Rejecting & $0.67^{*}$ & $0.56^{*}$ \\
\hline Object Punishment & $0.26^{* *}$ & 0.21 \\
\hline Demanding & $0.43^{* *}$ & $0.39^{* *}$ \\
\hline Indifferent & $0.45^{* *}$ & $0.53^{* *}$ \\
\hline Symbolic Reward & -0.19 & $-0.34^{* *}$ \\
\hline Loving & $-0.41^{* *}$ & $-0.40^{* *}$ \\
\hline Object Reward & -0.21 & -0.19 \\
\hline Neglecting & 0.24 & 0.27 \\
\hline
\end{tabular}

$* *$ Significant at .05 level of significance *Significant at .01 level of significance

In the above Table IV moderate level of neuroticism is considered in both adolescent males and females and correlation coefficient is computed for the ten dimensions of parent-child relationship. Significant positive correlations(low to moderate) were obtained for the parent-child relationship that are Indifferent, Using Object Punishment, Demanding and Rejecting for adolescent males and Indifferent, Neglecting, Demanding, Rejecting, using Symbolic Punishment for adolescent females. Protecting and loving parent-child relationships using symbolic rewards are negatively correlated with neuroticism in adolescent females. Researchers have indicated greater affiliative needs among females than in males. Significant negative correlation was obtained for the dimensions of parent-child relationship that are loving in adolescent males. Warmth and acceptance, as opposed to coldness and rejection, is probably the most influential of all aspects of child-rearing. These have significant implications for the socialization of the child. The above table IV indicates significant moderate positive correlation between neuroticism in both males and females who reported a rejecting relationship with their parents. Rejection is one of the major sources of delinquency especially rejection of boy child by his father (Michael Argyle, 1964) and is a source of neurotic tendencies. Neurotics are found to have been rejected or to have had conflict with their parents more than in normal. Thus the third hypothesis (H3) that there would be no 
significant correlations among pattern of parent-child relationship and neuroticism in adolescent girls and boys is rejected.

\section{Conclusion}

To sum up, the study examined all three hypotheses as proposed. Neuroticism is an important predictor of well being (Yeung, Hemsley, 1997). Gender differences in body self-image and in reactivity to interpersonal stress especially in the context of family are empirically established. Thus the above results and their discussion helped to examine the influence of perception of individual attractiveness and interpersonal relationship (with parents) on neuroticism in adolescent boys and girls and established significant correlations among the defined variables.

\section{Summary}

1. Analysis of data obtained confirmed the conclusions of other studies that there is a positive correlation between physical attractiveness and neuroticism in females.

2. An association was found between the level of parent-child relationship and neuroticism especially the dimensions of Rejection, Indifference, Demanding and Loving.

3. A negative correlation was computed between Neuroticism and Symbolic reward in adolescent females thus stressing the importance of psychological rewards and parental/interpersonal acceptance in females.

However the study is limited by the small sample size $(\mathrm{N}=100)$. Also the self-ratings of individual or personal attractiveness should be corroborated with the ratings by peers and significant others to provide better understanding. Interpersonal stress due to peer relations can be taken up in a further study.

\section{References:}

[1]. Burton Scot, Netemeyer, Richard, Lichtenstein, R.Donald (1995), Gender Differences in Appearance related Attitude and BehavioursImplications for Consumer Welfare, Journal of Consumer Research, Vol.22, No.3, pp.60-75.

[2]. Burgess, W.Earnest, Wallin, Paul (Feb.1944) Personal Appearance and Neuroticism as related to Age at Marriage, Human Biology, Vol.19, No.1, pp.15-22.

[3]. Devore, M.Dorothy (2006) Parent-Child Relations: New Research, Nova Science Publishers Inc., New York.

[4]. Eysenck, J.Hans (1991) Neuroticism, Anxiety and Depression, Psychological Inquiry, Vol.2, No.1, pp.75-76.

[5]. Henkin, L.Benjamin, Mermelstein, Robin, Roesch, Linda (2007), Sex Differences in Adolescent Depression: Stress Exposure and Reactivity Models, Child Development, Vol.78, No.1, pp.279-295.

[6]. Krishnaswamy, Saroja, Mantri, Pratima (1997), Psychological correlates of Marital Adjustment among Working Women, International Journal of Sociology of the family, Vol.27, No.1, pp127-138.

[7]. Moffit, F.Paul, Eisen,Peter (May1982), Factors correlated with Neuroticism Scores for Married Women, Journal of Marriage and Family, Vol.44, No.2, pp.491-496.

[8]. Robbins, W.Richard, Trzreniewski, Kali (2005), Self esteem-Development across Lifespan, Current directions in Psychological Science, Vol14, No.3, pp.158-162.

[9]. Reilly, Thomas, Greeves, Julie (2002), Advances in Sports, Leisure and Ergonomics, Routledge, New York.

[10]. Vitters, Joar, Nilsen, Fredrik (2002), The Conceptual and Relational Structure of Subjective Well-Being, Neuroticism and Extraversion: Once again Neuroticism is the important Predictor of Happiness, Social Indicators Research, Vol.57, No.1, pp.89-118. 